(I) Internal hydrocephalus.

The foramina in the roof of the fourth ventricle (see Fig. 7) may become blocked by adhesions, and the pressure rise being inside the ventricles cannot be measured by a spinal manometer. Theoretically there may be an obstruction of the narrow sylvian aqueduct, due to ependymitis. This certainly occurs apart from evident meningitis, and results in dilatation of the third and lateral ventricles only.

(2) Communicating hydrocephalus.

The ventricles communicate with the cisterna magna and the spinal theca, but there is an obstruction to the upward flow through the opening in the tentorium (incisura tentorii). As the greater part of normal absorption is above the tentorium a marked increase in pressure results, both in the ventricles and in the spinal subarachnoid space. Some cases of "idiopathic" hydrocephalus in children are probably of this type.

\title{
Lumbar Puncture.
}

The actual technique of lumbar puncture is too well known to need description here. The importance of ascertaining both the pressure and the composition of the cerebro-spinal fluid in every case suspected of meningitis or showing signs of meningism will be clear from the articles which follow. Whilst this is being taken, the patient's head should be kept. extended-not flexed on the chest; breathing should be quiet and regular; there should be nothing tight round the neck (a tight pyjama collar, or even a necklace, will in a strained position, run the pressure up appreciably); and there should be no undue abdominal compression by the patient's knees.

\section{SUPPURATIVE MENINGITIS}

\author{
By A. GURNEY YATES, M.A., M.D., F.R.C.P. \\ (Phys. Royal Infirmary, Sheffield; Lecturer in Medicine, University of Sheffield)
}

The term suppurative meningitis indicates that we are basing our classification on a particular type of meningeal reaction, namely, that in which the cerebro-spinal fluid shows a pleocytosis, wholly or predominantly polymorphonuclear, and to the naked eye is turbid or frankly purulent. This type of reaction is the result of infection with pyogenic bacteria: We are therefore dealing with a number of separate diseases, but as they are similar in many essential particulars it is both practical and convenient to class them together into a group, excluding, however, meningococcal meningitis, which it is usual to consider separately.

The bacteria most usually found are streptococci, staphylococci, pneumococci and, in children, B. "influenzae" (so-called). Many others are encountered occasionally, including gonococci, B. typhosus, B. paratyphosus, B. coli, pneumobacilli and anthrax.

Suppurative meningitis as thus defined is usually secondary to some focus of infection elsewhere, but this is not invariable. Pneumococcal and so-called "influenzal" (Hæmophilus) cases, and occasionally others maiy be primary.

\section{Source of infection.}

\section{Suppurative otitis media.}

Something like 50 per cent of cases are secondary to suppurative otitis media, acute or chronic. Otologists have studied in great detail the exact paths of invasion. We need not here concern ourselves with the minutiae of this process; a broad outline will suffice. The spread may occur directly by the erosion of bone, or along vascular, usually venous channels by septic thrombosis: The direction of spread may be backward, via the mastoid antrum, or inward, causing a labyrinthitis, and spreading thence via the internal auditory meatus, or the aqueduct of the cochlea to the posterior fossa, or upward through the roof of the tympanum to the middle fossa. The process of spread, however, is not always as immediate and direct as this. An 


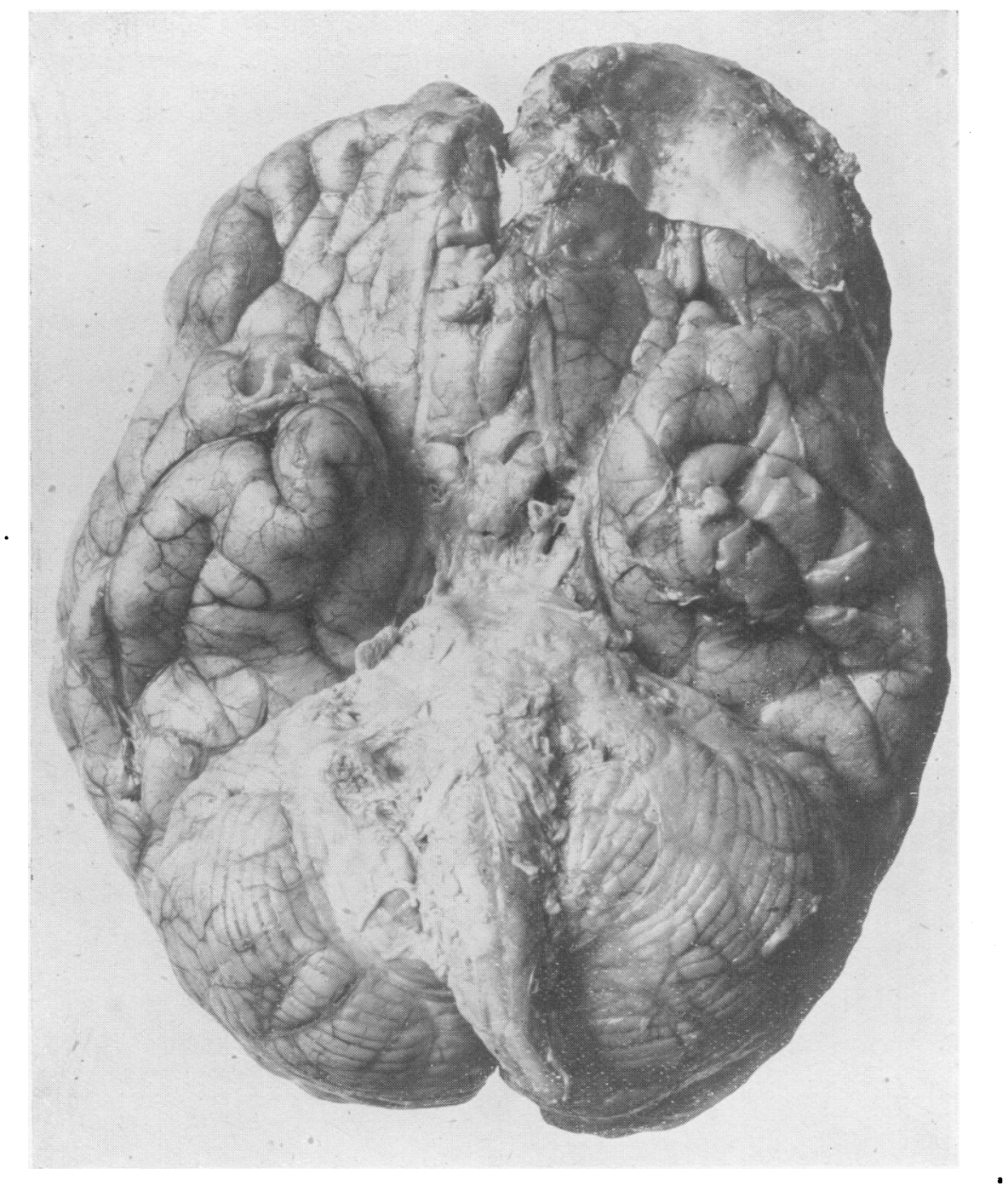

FIG. 9.-Acute post-basic meningitis due to the spread of a Staphylococcus aurens infection from an oldstanding suppuration of the left frontal sinus in a man, aged 29 years, who had suffered from fracture of the skull seven years previously. A chronic extradural abscess followed, and then several chronic abscesses in the frontal lobe (see Fig. Io). One of these invaded the lateral ventricle, with rapid acute spread downwards (Fig. 7) within the brain to the base (see pp. 7I-2). (From Beattie and Dickson's Textbook of Pathology, Fig. 643.) 


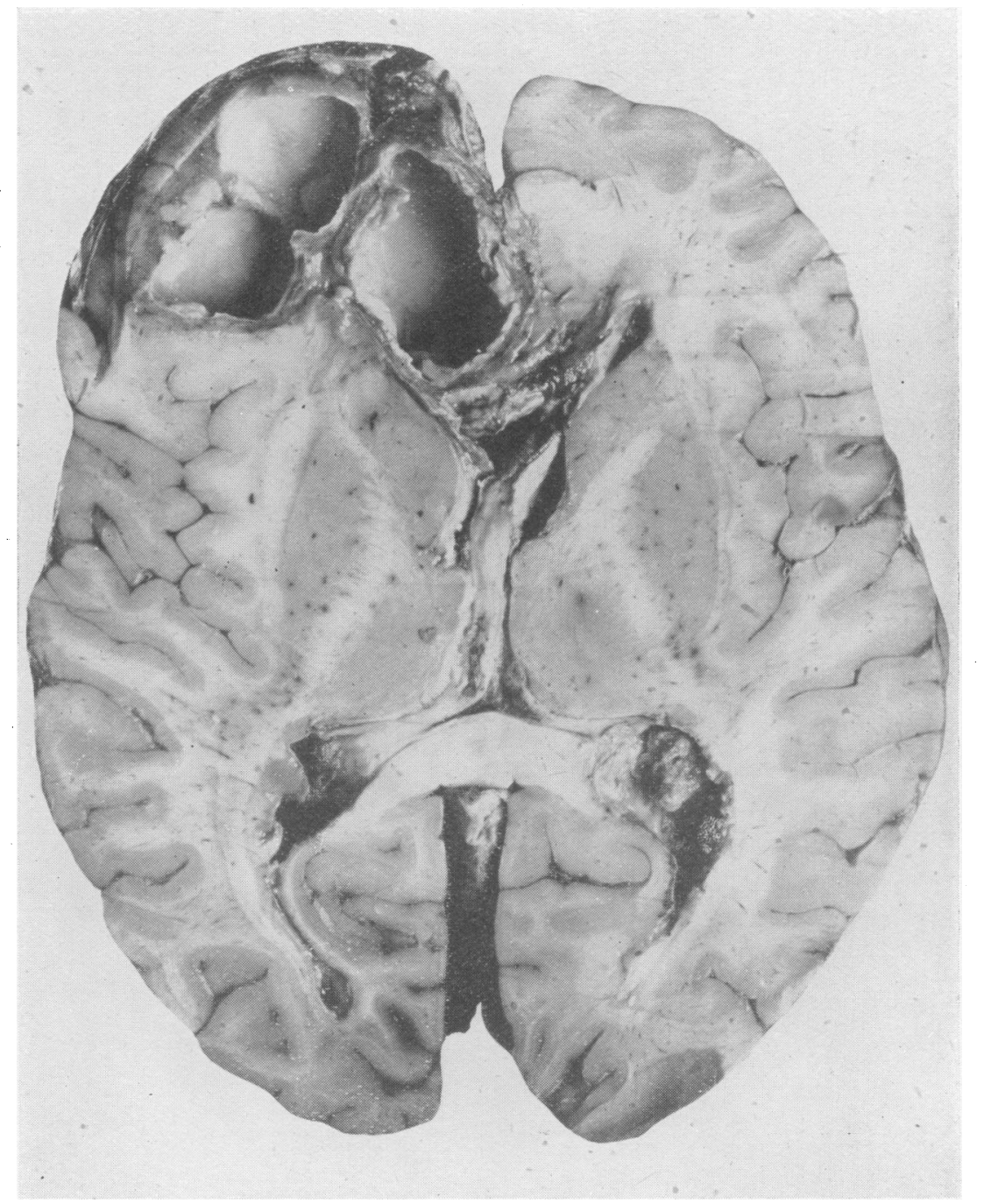

FIG. 'Io.- Horizontal section through the cerebral hemispheres of the same case, showing the chronic abscesses in the left frontal lobe, with invasion of the lateral ventricles and a terminal acute spread downwards as shown in Fig. 9, to the base of the brain. (Beattie and Dickson's Textbook of Pathology, Fig. 643B.) 
infected middle ear may cause first a cerebral abscess or a sinus thrombosis, which in turn may infect the meninges.

\section{Paranasal sinus infection.}

In acute or chronic paranasal sinusitis the infection may spread to the subarachnoid space either directly through the bony wall of the sinus, or by venous channels. The maxillary antrum is the least prone of all the sinuses to lead to intracranial complications.

\section{Septic lesions in the neighbourhood.}

Such lesions are always potentially dangerous. Throat infections, orbital suppuration, erysipelas or other septic lesions of the face, osteomyelitis of the facial or cranial bones, and carbuncles are all capable of causing meningitis.

\section{Trauma.}

Compound fractures of the skull may open up a direct path of infection, especially if thedura is torn, and it is important to remember that a fracture passing through the wall of a paranasal sinus, or through the petrous temporal bone into the middle ear, is essentially a. compound fracture. It has recently been pointed out that such fractures often fail to heal by bony union, so that a channel of infection may persist, and cases have been reported of meningitis infected via such traumatic channels months or years after the injury (see Figs. 9: and Io).

\section{Distant Foci.}

Infection from distant lesions is generally carried by the blood stream. The most important causes in this group are acute and chronic pulmonary diseases, pneumonia, lung abscess, bronchiectasis, general blood infections, septicaemia, pyaemia and malignant endocarditis.

\section{Pathology}

When pyogenic bacteria gain access to the subarachnoid space a very dangerous situation ensues, because the invading hosts, which have penetrated the defences, can at once fan out and occupy with little opposition a vast territory. This territory is the whole of the immensely complex subarachnoid space, to any and every part of which the invader can readily gain access. This catastrophe occurs the more readily because the organisms are caught up in the slowly flowing stream of cerebro-spinal fluid, as it passes from the choroid plexuses, out to the basal cisterns, and upwards to the convexity, to be absorbed by the arachnoid villi. This fluid, moreover, which carries the infection, possesses very feeble anti-bacterial properties, so that the organisms have things very much their own way. This dissemination can take place rapidly and leads to the characteristic pathological feature, a purulent exudate in the subarachnoid space (see Figure 9). The pia-arachnoid is at first red and congested, and the turbid exudate later becoming purulent, spreads along the sulci and may form a continuous film on the surface of the brain. It may be most pronounced over the vertex as in pneumococcal meningitis, or at the base where it overspreads the cranial nerves with results which influence the clinical picture. Downward spread over the cord may also occur.

The subjacent brain may be unaffected, or merely flattened by increased pressure, but. sometimes the surface is congested and there is a superficial encephalitis. Veins on the surface may be thrombosed.

Adhesions are apt to form around the fourth ventricle which interfere with the normal flow of cerebro-spinal fluid to the surface. Thus the ventricles become distended, and internal hydrocephalus is the result. In cases where the convexity is severely affected absorption by the arachnoid villi may be hindered. As a result, therefore, of the copious exudate, combined with an impeded outflow and absorption of cerebro-spinal fluid, a considerable increase in intracranial pressure results.

\section{SYMPTOMS AND SIGNS}

The onset is often sudden and may be fulminating; on the other hand it may be slower, and characterised for a short time by a general malaise. It is never gradual and insidious, 
but when secondary to some other acute infection the initial phase may be obscured by symptoms already present.

The fever, often high and continued, may be accompanied by rigors. The pulse in the early stages may be relatively slow. In children convulsions are frequent.

The meningitic syndrome which is common to all varieties is the result of widespread meningeal irritation brought about by toxic action and increased tension. In infants the fontanelle may bulge.

The patient lies curled up in bed resentful of all stimuli. Photophobia is frequent. Headache is constant, and is something more than the headache of a feverish state. Its intensity is such that in children it sometimes leads to a piercing hydrocephalic cry. It is not as a rule localised, and the pain may radiate down the spine. Cerebral vomiting is frequent. The general irritability and restlessness is accompanied by delirium; the mind becomes clouded and stupor merges into coma. There may be retention or incontinence of urine. The reflexes are inconstant, but, as in most cases in which intracranial pressure has risen rapidly, they tend to be subnormal. The plantar reflexes are usually flexor, but may be extensor in the later stages. The abdominal reflexes are usually absent, and the abdomen scaphoid.

The meningeal irritation leads to certain very characteristic physical signs; neck rigidity, Kernig's and Brudzinski's signs. Passive flexion of the head on the chest causes spasm of the neck extensors. The resistance can at once be felt and the facial expression indicates that it is painful. Head retraction in which the head is bent back in rigid extension is an extreme degree of the same thing. Kernig's sign is a painful spasm of the hamstrings elicited by first fully flexing the hip and then attempting to extend the knee. On flexing the head-as in testing for neck rigidity - the hips and knees flex (Brudzinski's neck sign). On passive flexion of one leg on the abdomen the other leg flexes similarly (Brudzinski's leg sign).

Cranial nerve palsies are frequent in basal cases. Ptosis, squint, diplopia and pupillary abnormalities, especially inequality and sluggishness to light, are frequent. The optic discs may be congested; frank papilloedema is uncommon. Other cranial nerve lesions are less frequent, but facial paralysis may occur and sometimes infection spreads along the internal auditory meatus to the labyrinth, causing vertigo, nystagmus and total deafness on the affected side. Limb paralyses are uncommon except for the general flaccid paralysis of the final stage, but a mono- or hemi-plegia may be due to involvement of the cerebral cortex.

\section{The Cerebro-Spinal Fluid}

When meningitis is suspected a lumbar puncture should be done without delay. If, as very occasionally happens, fluid cannot be obtained by the lumbar route, a cistern puncture should be done. The fluid is under increased pressure, turbid or purulent, and may be tinged with yellow or yellowish green, A fibrinous coagulum is sometimes seen. Exceptionally in the early stages the fluid may be clear, but an increase of cells will still be found.

The cell count is always increased and may reach many thousands per c.mm., and on staining the majority are polymorphonuclear, but a proportion may be lymphocytes. The more acute and severe the case, the more likely are the cells to be all or nearly all polymorphs. In recovering and less acute cases the proportion of lymphocytes is usually higher. Bacteria may be recognised in appropriately stained films and grown on suitable media. Sometimes, however, a purulent fluid may prove sterile on culture.

The normal protein content of $15-40 \mathrm{~m} . \mathrm{g}$. per Ioo cc. may be increased tenfold or more, the globulin also is raised. The sugar is reduced and may be absent. This is said to be due to its being used up by the invading bacteria. The reduction of chlorides is an important sign. It is mainly due to an increased permeability of the membranes, and affords some indication of the degree and extent of their involvement. The normal is $720-750 \mathrm{mg}$. per roo c.c., and they may fall to 650 or less, but readings below 600 are usually due to tuberculous meningitis. The colloidal gold curve is in the meningitic zone. Complement and haemolysin can usually be found.

\section{Diagnosis}

Signs of meningeal irritation occur in a number of conditions which must be distinguished from true suppurative meningitis. In acute fevers, and especially in pneumonia, severe head- 
ache, cervical rigidity, and a positive Kernig may occur, but the cerebro-spinal fluid is normal. This condition is known as meningism, and is probably toxic in origin.

In poliomyelitis and polioencephalitis the clinical signs of meningeal irritation often occur at the outset, but the distinctive paralyses quickly follow. The cerebro-spinal fluid is never purulent. There may be a considerable number of polymorphonuclear cells in the early stages, but the chlorides and glucose are normal.

In the various forms of meningo-encephalitis there are signs of early damage to the central nervous system, and the cerebro-spinal fluid is generally normal, or if there is a pleocytosis it is lymphocytic.

Spontaneous subarachnoid haemorrhage has a typical sudden onset, with pain as a rule at the back of the head, and neck, vomiting, and often rapid loss of consciousness. The cerebrospinal fluid is uniformly blood-stained, and, on centrifuging, the supernatant fluid is tinged with yellow.

In tuberculous meningitis the onset is insidious. The cerebro-spinal fluid is usually clear to the naked eye, the cells showing a varying proportion of lymphocytes. An extremely low chloride is significant, and in a considerable number of cases tubercle bacilli can be demonstrated.

The diagnosis of meningococcal meningitis rests upon the isolation of the specific organism Acute syphilitic meningitis can simulate the pyogenic form. The pleocytosis is lymphocytic, but occasionally polymorphonuclear. In such cases the Wassermann will decide.

A cerebral abscess may or may not be febrile, but unless it is in a silent area focal symptoms will point to a definitely localised lesion. The cerebro-spinal fluid may be normal, but there is often a slight or moderate mixed cellular increase. It is only in exceptional cases that the number of polymorphs could simulate a suppurative meningitis, but unless a meningitis actually coexists the chlorides and glucose will be normal.

In cerebral sinus thrombosis the temperature is of the swinging hectic type associated with rigors. There are often external signs such as localised oedema and tortuous dilated veins to indicate the site of the thrombosis. The cerebro-spinal fluid changes are similar to those in cerebral abscess.

When pyogenic bacteria actually invade the subarachnoid space and set up a meningitis there may still be great variations in the effects produced, due to differences in the degree and extent of the invasion. Exceptionally the fluid may be clear to the naked eye, and only a moderate polymorphonuclear reaction found. This may be a very early stage of a virulent pyogenic infection, before the chlorides or glucose show much change or the proteins have risen. It is in such cases that the complement and haemolysin tests are valuable. Their presence with a normal protein would be of serious omen.

Usually, however, the fluid is turbid, but the number of cells gives no certain indication of the extent or severity of the meningeal involvement. If few or no organisms are found, cultures are sterile and chlorides normal, it is likely that the condition is due to a localised focus which is pouring out cells without causing widespread infection of the meninges. An infected ear or air sinus, a cerebral or extra-dural abscess, or a patch of meningitis which has localised itself can do this. If the primary cause can be dealt with this form is often benign, but it may be a prelude to a more widespread virulent infection of the meninges.

\section{Prognosis}

Favourable features: a primary cause that can be dealt with; few or no bacteria in the fluid; sterile cultures and normal chloride. chloride.

Unfavourable features: A primary cause difficult to deal with; positive cultures, low

The prognosis depends to a considerable extent upon the kind of organism present. Infections with pneumococci, staphylococci, and in infants B. "influenzae" are particularly grave, but with the development of chemotherapy much depends upon the susceptibility of the organism to the various members of the sulphonamide group of drugs.

\section{TREATMENT}

The first thing to do in the treatment of suppurative meningitis is to diagnose it early, and as it is usually secondary to some pre-existing infection the next thing is to find the primary focus and deal with it. This is particularly imperative where the meninges are being infected 
by a localised focus of pus under tension. If no such focus is obvious a careful search should be made. Prompt surgical treatment may avert a meningitis before it is well established. Pus in a mastoid, for example, may lead to symptoms of meningitis with a purulent or turbid fluid before living organisms can be detected in it, and in such cases prompt drainage may be followed by quick recovery. In well-established cases prompt operation is no less important.

The withdrawal of cerebro-spinal fluid by lumbar puncture is a method of treatment as. well as a means of diagnosis. It relieves headache by lowering tension, removes toxi-infective material, and affords valuable evidence on which to judge the progress of the case. It should be carried out daily at first, and enough fluid should be removed to bring the pressure approximately to normal. At the same time fluids should be given freely by mouth. In certain cases an intravenous saline drip is very useful.

Attempts have been made to drain the subarachnoid space by leaving a needle in situ for a time, but it is difficult to carry out.

Ayer introduced a method of lavage by doing a cisternal and lumbar puncture at the same time, and washing through from above downwards with saline. It has not, however, come into general use and is not without danger.

The sulphonamides have improved the outlook even in this most deadly form of meningitis, and administration should be started at once, because during the stage of acute invasion the organisms are multiplying with amazing rapidity, and the greater the number of organisms the less effective is the drug. It is also evident that the sooner the growth of bacteria can be checked the less will be the tissue damage. It is also important to commence with an effective dose which will promptly produce in the blood and cerebro-spinal fluid an adequate concentration. If the initial dose is inadequate there is danger that instead of stopping their growth it will have the effect of making the organisms more resistant.

Choice of drug.- The most active drug available should be chosen without waiting for the organism to be identified. Sulphapyridine is more active than sulphanilamide, and sulphathiazole more so than either. Sulphathiazole or sulphapyridine are therefore the drugs of choice. The newer sulphamezathine, however, has several advantages over the others, and is said to be equally potent and less toxic. It is more soluble, and therefore less likely to cause renal damage and being more slowly excreted it can be given six hourly instead of four hourly.

Dosage.-It is an advantage to give the initial dose intravenously, which for adults should be 2-4 gm., according to weight. This should be followed by I. $5 \mathrm{gm}$. four hourly for four days, I gm. four hourly for two days, and I gm. six hourly for two days. For children the initial dose is $0.5^{-2}$ gm., according to age. Relapses are common, and it is advisable to continue small doses for several days after the temperature is normal.

In order to ensure a prompt and adequate blood concentration an estimation of the bloodlevel I2-24 hours after the beginning of treatment is a valuable precaution. If it is less than 3-4 mg. per Ioo cc., and the patient is gravely ill, an additional intravenous dose can be given. In view of the large doses the usual precautions must be observed, especially the administration of large amounts of fluid and examination of the blood.

Symptomatic treatment consists of the relief of restlessness and headache. For the former full doses of bromide and chloral or phenobarbitone may be used, and for the latter morphia.

Penicillin.-This drug is not yet available, but recent reports suggest that when it is, we may have a means of treatment possibly surpassing anything yet achieved. $\mathrm{H}$. W. and M. S. Florey report amongst other cases one of staphylococcal cavernous sinus thrombosis, and one of streptococcal meningitis, which recovered after treatment with penicillin after having proved refractory to the sulphonamides.

There is some difference of opinion as to the value of antisera as an adjunct to the sulphonamides. In most varieties of suppurative meningitis it is probably useless, but in the pneumococcal form it may be of value if the organism can be typed and a specific serum obtained. Thomas and Twort have reported the case of an airman who made no progress on sulphapyridine alone, and was desperately ill, and apparently moribund. He improved dramatically after receiving 200,000 units of type 3 specific serum intravenously over a period of two days, and his life was saved. Leyshon reports six cases with five recoveries in which sulphapyridine was supplemented with specific antiserum intrathecally.

Influenzal meningitis is not a common variety, and occurs most frequently in infants, in whom it is almost invariably fatal. The ventricles may be full of pus, and loculation and obstruction are prone to cause internal hydrocephalus. On the whole sulphonamide treatment 
has not proved very successful, but Knouf and others report I2 cases with 9 recoveries, 5 of whom were under two years of age. They were all treated with a continuous drip of sodium sulphapyridine, 0.5 or I per cent in normal saline. Sako and others also report 7 cases under four years old with 5 recoveries. These cases were treated with large doses of sulphadiazine orally. A total of 80 to $90 \mathrm{gm}$. of the drug was given to children aged 2 to 3 years, and the drug was given in reduced doses after apparent cure for at least two weeks. The comment of Dr. Stanley Banks in the Medical Annual is worth quoting. "These papers provide a valuable pointer to the intensity and duration of the sulphonamide treatment necessary for success in this dire disease. Treatment on the ordinary lines appropriate for meningococcal meningitis has shown many failures."

\title{
REFERENCES
}

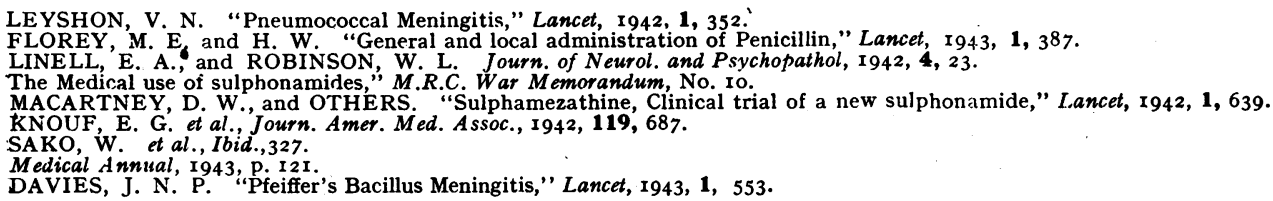

ENCEPHALITIS

\author{
By LEWIS R. YEALLAND, M.D., F.R.C.P. \\ (Phys. Prince of Wales Hosp. and West End Hosp. for Nervous Diseases, etc.)
}

Inflammation of the brain may occur as a primary disease, but it is most frequently due to an extension of infection from other parts. A combined inflammation of the brain and spinal cord may occur, giving rise to a condition which is called encephalo-myelitis. The lesion of poliomyelitis is not always limited to the spinal cord, it sometimes extends into the brain stem or farther up into the brain. This is called polioencephalitis superior. Encephalitis may result from an extension of disease, (a) from the meninges in tuberculous, epidemic or other form of meningitis, meningo-encephalitis; (b) from the aural or nasal sinuses, or from bronchiectasis, suppurative encephalitis (abscess of the brain); and from (c) injuries of the skull, traumatic encephalitis. Further, encephalitis may occur as a complication of the specific fevers, and, in particular measles and scarlet fever; and vaccination. Finally, disseminated patches of encephalitis are to be found sometimes in syphilis when there is no evidence of that disease elsewhere.

Particular attention will be directed here to the following four types of non-suppurative encephalitis, all of which may be regarded as rare: (i) Encephalitis lethargica. (ii) Encephalitis periaxialis. (iii) Acute disseminated encephalomyelitis. (iv) Otogenic encephalitis. The first three of these are primary; the last named is secondary to infection in the ear.

\section{DIAGNOSIS OF ENCEPHALITIS}

In the early phases of encephalitis there may be general symptoms of pyrexia, headache, irritability, convulsions, delirium and vomiting. On the other hand, there may be coma more or less profound. Neurological signs do not, as a rule, make their appearance during the first day or two of the disease, and the condition is often called influenza if no focus of infection can be discovered. Indeed the acute infectious initial phase tends to mask the neurological features. Moreover, when intracranial localising signs are brought to light, the acute infectious phase may have passed off and such indications of intracranial disease as, for example, hemiplegia or aphasia may suggest other causes than that of encephalitis. In these circumstances difficulties in diagnosis arise, not as to where the lesion is situated, but as to what is its 